\title{
Naturwissenschaft, Technik und sprachliche Bildung in Afrika
}

\author{
Von Eckhard Breitinger
}

Seit der UNESCO-Konferenz in Addis Abeba 1961 hat sich eine lange Reihe ministerieller und internationaler Konferenzen damit befaßt, die bildungs- und entwicklungspolitischen Zielvorstellungen der afrikanischen Länder zu definieren und Wege zu ihrer Realisierung zu weisen. Die Deklarationen und Empfehlungen, die von diesen Konferenzen ausgesprochen wurden, zeigen sowohl die Konstanten der bildungs- und entwicklungspolitischen Zielsetzungen wie auch die Variablen, d. h. die auf Lern- und Erfahrungsprozessen gründende Anpassung an die tatsächlichen und keineswegs allzu ermutigenden Entwicklungen in Afrika. Die durchgängige Konstante von Addis Abeba 1961 bis zur Harare-Konferenz in Zimbabwe im Juli 1982 ist die Hoffnung, über eine Offensive im Bildungswesen die wirtschaftliche und soziale Entwicklung in Gang zu setzen und zu beschleunigen. In Addis Abeba wurde ganz auf quantitatives Wachstum gesetzt: allgemeine Schulpflicht und breite Alphabetisierung sollen wirtschaftliches Wachstum, gedacht im Sinne industrieller Entwicklung, nach sich ziehen. Bereits bei der LagosKonferenz von 1964 verschob sich der Akzent vom rein quantitativen auf ein qualitatives Wachstum. Gefordert wurde die Erforschung und Nutzbarmachung der spezifisch afrikanischen Ressourcen und die Afrikanisierung der Lehrbücher, d. h. Anpassung der Lehrmaterialien an die afrikanischen Ressourcen, an die geographischen und demographischen Gegebenheiten. Mit Nairobi, Monrovia, Accra, Dakar und Lagos folgten Forderungen nach Verlagerung des Schwerpunktes auf Naturwissenschaft und Technik, Land- und Hauswirtschaft, nach Höherbewertung der af rikanischen kulturellen Traditionen, nach Einführung der afrikanischen Sprachen als Unterrichtsfach oder Unterrichtsmedium, nach naturwissenschaftlichem Basisunterricht in den Grundschulen, ${ }^{1}$ also ein Schwanken zwischen Human- und Naturwissenschaft, zwischen Kultur- und Verwertungswissenschaften. Wie ich im weiteren zu zeigen hoffe, ist dieses Schwanken jedoch nicht - zumindest nicht gänzlich - auf Unentschiedenheit in der bildungspolitischen und pädagogischen Zielsetzung zurückzuführen, sondern reflektiert die Lern- und Afrikanisierungsprozesse in der curricularen und didaktischen Umsetzung der beschriebenen Zielvorgaben in die Schul- und Ausbildungspraxis. Dies wird an der Deklaration von Harare deutlich: In der Bestandsanalyse der Harare-Deklaration wird auf die Ineffizienz und Unwirtschaftlichkeit des formalen Bildungssystems verwiesen, weil es den Bedürfnissen und Gegebenheiten nicht genügend Rechnung trägt:

1 Vgl. F. P. Nalletamby: Science and Technology Eduation for Development in Africa, EDUCAFRICA 7 (1981), 104 S. u. Baba Akhib Haidara, The Harare Conference: Meaning and Significance of an Event, EDUCAFRICA Special (1983), 7-10. 
The high rates of student wastage through drop-outs and repeats, which are a cause of illiteracy and which reduce the intake capacity of education systems, are both social and educational in origin. Often, it is the very conception of eduction, its aims and content, its structure, the values that it instils and the spirit in which it operates that are at issue.

Um dem zu begegnen, werden $u$. a. folgende Ziele formuliert:

- to improve the command of up-to-date knowledge, particularly scientific and technological knowledge, by strengthening basic and applied research capabilities and training potential, and through a judicious use of existing scientific and technological knowledge in order to further economic and social progress;

- to develop and renovate science and technology teaching at all levels and activities designed to popularize science among the general public, in order to promote full utilization of modern scientific and technical knowledge in development ...;

- to restore to their rightful status the African heritage and the traditional social and human values that hold potential for the future and for progress;

- to develop the use of African or national languages as languages of instruction, taking the necessary steps to that end with regard to research, training of staff and production of material; ${ }^{2}$

Es ist keineswegs zufällig, daß hier die Vermittlung von Kenntnissen in moderner Wissenschaft und Technologie angebunden wird an die Darlegung vorhandener, sprich afrikanischer Kenntnisse in Wissenschaft und Technologie. Das Vertraute - die traditionellen Technologien, die das Tor zu dem Neuen, Unbekannten - den modernen Technologien - öffnen sollen, sind eben ein Teil jenes kulturellen Erbes, das es zu pflegen gilt, das über den Gebrauch der einheimischen Sprache zu pflegen ist, die ja ein Teil dieser Tradition, zugleich aber auch Träger und Vermittler dieser Tradition ist. Wenn es gelingt, von der traditionellen Technologie auf moderne Technologien auszugreifen und dabei das Medium der afrikanischen Sprache beizubehalten, dann müßte es auch eher gelingen, der modernen Wissenschaft und Technik den Charakter des Fremdartigen, den Charakter eines unangepaßten und unanpaßbaren Fremdkörpers zu nehmen. Angepaßte Technologie, die wirklich angepaßt sein will, kann eben keine reduzierte Technologie sein, die von technologischen Alleskönnern partriarchalisch auf das Niveau von bescheidenen Geistern zurückgestutzt wird - damit eben wieder nur Fremdbestimmung in gefälligerem Kleid ist -, sondern angepaßte Technologien müssen sich die Abnehmer selbst anpassen, indem sie sie sich aneignen, Besitz von ihr ergreifen, durch Anpassung vor allem über kulturelle und soziale Akzeptanz. ${ }^{3}$

Einige Bemerkungen zur jüngeren Entwicklung des formalen Bildungssystems in Afrika können sowohl die Schwächen der bisherigen naturwissenschaftlich-technischen Ausbil-

2 The Harare-Declaration, EDUCAFRICA Special (1983), 33-38; hier 35, 36/37.

3 Vgl. Souleymane Bachir Diagne, Science: The Stranger in Our Midst, Vortrag bei UNESCO-Konferenz Comparative Philosophical Studies on Changes in Relations between Science and Society, Kingston, Jamaica, 21-24 Juni (1983). 
dung, wie auch die späte aber unentbehrliche Verquickung von Erziehung zum naturwissenschaftlich-technischen Verständnis und von Erziehung zur Sprachlichkeit erläutern. Die Missionsschulen, wie die öffentlichen Schulen der Kolonialzeit, hatten kulturmissionarische Zielsetzungen und dienten der Ausbildung einer schmalen Elite von zukünftigen Verwaltern. Die Ausrichtung war daher überwiegend geistes- und sozialwissenschaftlich. Kirche und Kolonialverwaltung haben in der Vergangenheit das formale Bildungswesen kontrolliert, das den kulturellen und sozialen Wandel der vorkolonialen afrikanischen Gesellschaften am nachhaltigsten beeinflußt hat. Aber gerade diese beiden Institutionen haben gesellschaftliche und politische Zielsetzungen vertreten, die, wenn sie in pädagogische Praxis umgesetzt werden, wissenschaftliches Denken eher behinderten als förderten.

Für die Mission ist das erste Erziehungsziel die Lesefähigkeit für die Bibel, d. h. für einen religiösen Text, der als solcher nicht zur Disposition für kritisch-analytisches Infragestellen steht. Die Kolonialverwaltung hatte als Ausbildungsziel das Training von Administratoren in den mittleren Chargen, die die Fertigkeiten des Lesens, Schreibens, Rechnens, Buchführens beherrschen, aber nicht das System in Frage stellen sollten. Für die beiden Institutionen, die das Schulwesen kontrollierten, war das fraglose Hinnehmen der gegebenen politischen Situation wichtigster Teil des "heimlichen Lehrplans", damit Rezeptieren, Memorieren, Reproduzieren die angemessene Unterrichtspraxis. Faktendrill und Fertigkeitendressur haben und sollten die Fähigkeit zu Transferleistungen, zum Umsetzen von Fertigkeiten in andere Bereiche, wo sie emanzipatorisch wirksam werden könnten, verhindern. Und wo die Bewältigung und Veränderung der materiellen und politischen Umwelt nicht Ziel der Bildung war, konnten auch nicht die pädagogischanthropologischen Voraussetzungen geschaffen werden für die zweite wichtige Phase des Wandels in den afrikanischen Gesellschaften, des Wandels in Richtung auf wieder autonome und auch wissenschaftlich operierende Gesellschaften. So verfügte etwa Nigeria mit seinen damals rund 60 Millionen Einwohnern am Tag der Unabhängigkeit lediglich über eine universitäre Ausbildungsstätte in Ibadan, ein College der University of London mit Liberal Arts College-Zuschnitt mit einigen hundert Studenten. Erst mit der Unabhängigkeit wurde die University of Nigeria/Nsukka gegründet, mit naturwissenschaftlich-technischer Ausrichtung und einer pragmatisch orientierten Curriculumspolitik nach amerikanischem Muster. ${ }^{4}$ Heute hat Nigeria rund 20 Universitäten und eine Studentenbevölkerung von 80000 auf ca. 80 Millionen Einwohner, wobei die Neugründungen der letzten Jahre überwiegend Colleges of Science and Technology sind. Mit der Unabhängigkeit wurden die Lehrpläne in den geisteswissenschaftlichen Fächern am zügigsten afrikanisiert, denn dort war die Unangepaßtheit der aus europäischen Lehrplanschmieden stammenden Studiengänge am offenkundigsten. Die naturwissenschaftlichen Fächer jedoch blieben lange Zeit unangetastet, da sie unter dem Signum der reinen, wertfreien Wissenschaft als kulturneutral liefen. Viele der heute noch gültigen Lehrpläne wurden außerhalb Afrikas entwickelt, ebenso die Lehrbücher und Unter- 
richtsmaterialien. ${ }^{5}$ Das gängige Mißverständnis von Schulbildung als abstrakter Berechtigung für den Zugang zu weiteren Bildungs- und Aufstiegschancen, nicht aber als praxisorientierte Einrichtung, die verwertbare Fertigkeiten vermittelt, material- oder theorielastige Lehrpläne und eine auf Drill, auf reines Reproduzieren angelernter Fakten ausgerichtete Unterrichtspraxis wirkten zusammen, um Naturwissenschaften zu den unbeliebtesten Fächern zu machen. Nur $20 \%$ der Schüler behielten Naturwissenschaften am Ende der ersten drei Jahre Secondary School noch bei. ${ }^{6}$

Wenn man zu der Unangepaßtheit der Lehrpläne und der Erschwernis des Unterrichts in der fremden Sprache noch fachliche und sprachliche Schwächen der Lehrer einrechnet, so ist hier der Mißerfolg des Unterrichts vorprogrammiert. Wenn der naturwissenschaftlich-technische Unterricht eine so fremde Konzeptualität und Begrifflichkeit darstellt, ist es didaktisch-methodisch grundfalsch, zwischem dem Schüler und dem ohnehin fremden Unterrichtsgegenstand noch zusätzliche Barrieren in Form der fremden Unterrichtssprache Englisch oder Französisch aufzubauen. Beim Unterricht in der nichtafrikanischen Sprache wird vom Schüler ein doppelter Umsetzungsprozeß gefordert: Er muß seine Beobachtung des naturwissenschaftlichen Experiments in eine verbalisierte Begrifflichkeit umsetzen und diese Begrifflichkeit aus einem fremden, nur unzureichend beherrschten Sprach- und Vorstellungssystem in eigene, muttersprachlich gefaßte Vorstellungen und Konzeptionen umsetzen. Erst dann, nach der Umsetzung in die Muttersprache, ist kognitives Verstehen und Abspeichern ins Gedächtnis, d. h. der eigentliche Lernprozeß, möglich. Erschwerend kommt hier noch dazu, daß sich ein typischer Ubertragungseffekt von einer Sprache in die andere einstellen wird: Bei der Ubersetzung eines Begriffes, einer Konzeption aus der Ausgangs- in die Zielsprache, wird jeweils ein weiterer, umfassenderer Begriff in der Zielsprache gewählt. Es sei denn, es gäbe eine direkte Korrespondenz zweier Begriffe, was man aber bei europäisch-afrikanischer Ubersetzung wohl weitgehend ausschließen kann. Der weiter gefaßte Begriff bedeutet aber zugleich, daß die Begrifflichkeit an Schärfe und Prägnanz einbüßt. Der dargestellte wissenschaftliche Sachverhalt wird diffus, damit weniger verständlich. Der Lernende tut sich schwerer, von dem in sprachlicher Unentschiedenheit zerfließenden Sachverhalt geistigen Besitz zu ergreifen und sich ihn als Verhaltensrepertoire eigener wissenschaftlicher Kompetenz anzueignen.

Es ist offenkundig, daß in dieser pädagogischen Situation, wo sich das aus der Kolonialzeit überkommene und übernommene Erziehungssystem als eine Reststruktur (post)kolonialer Fremdbestimmung und Entfremdung erweist, der Erziehung zur Sprachlichkeit, der Sprachpolitik und Sprachplanung besondere Aufmerksamkeit geschenkt wird. Wiederum ist dieses Bewußtsein in den Geisteswissenschaften, speziell in der Literatur, am frühesten zum Ausdruck gebracht worden, einfach weil es dort so

5 D. Fall, Curcent Trends in Physical Science Teaching in Senegal, EDUCAFRICA, 7 (1981), S. 116; F. P. Nalletamby, Science and Technology Education, EDUCAFRIKA 7 (1981), S. 107.

6 Juliet I. Macauley, Teaching Foreign and Second Languages to the Very Young; Paper presented to the WAMLA-Conference on the Teaching of Foreign Languages in Ibadan, August 24-29, 1981. 
offenkundig ist. Chinua Achebe, der nigerianische Romancier, hat bereits in den frühen 60er Jahren gesagt, daß er sein Sprachmedium Englisch den nigerianischen Verhältnissen anpassen müsse, damit es seine Werthaltungen, Einstellungen, die spezifische kulturelle und soziale Atmosphäre seines Volkes, der Igbo, adäquat zum Ausdruck bringen könne. Achebe schreibt aber weiterhin in Englisch, das er in der Diktion, im Stil, vor allem durch die Verwendung von Ibgo-Sprichwörtern und Sentenzen anreichert, also durch die Verwendung von Elementen aus der Volkssprache und Volkskultur. Der kenianische Autor Ngugi wa Thiong'o hat 1983 in einem Vortrag in Bayreuth seine Beurteilung der Sprachenkonstellation in Afrika dargelegt:

I strongly feel that language is at the heart of the tension between the struggling cultures of dominated peoples of Africa and the agressive dominating cultures of imperialist nations. It is a well know fact of history that all the colonizing nations of Europe imposed their languages on the colonized peoples. Language is a carrier of a people's culture. Culture is a carrier of people's values. Values are a carrier of people's outlook or consciousness and sense of identity. ${ }^{7}$

Ngugi wa Thiong'o hat aus dieser Einsicht für sich als Schriftsteller eine radikale Konsequenz gezogen: Er schreibt nicht mehr in Englisch, sondern in seiner Muttersprache Kikuyu und ist erfolgreich damit. Erfolgreich bei einem breiten Publikum, das früher wenig oder gar nicht gelesen hat. Kritisiert wird er dagegen von dem elitären Lesepublikum früherer Jahre. Ngugi stand vielleicht mit seiner Hinwendung zur afrikanischen Sprache 1977 an der Spitze eines neuen Bewußtseins; mag sein, daß die Minister für Erziehung und Entwicklung 1983 in Harare eher die Nachhut dieser Bewegung bilden. Aber bei aller Unterschiedlichkeit der politischen Anschauungen zwischen Ngugi wa Thiong'o und den afrikanischen Ministern scheint die Deklaration von Harare mit ihrer Empfehlung, die afrikanischen Sprachen auch für den naturwissenschaftlichtechnischen Unterricht in der Grundschule als Unterrichtssprache einzuführen, doch von demselben Bewußtsein getragen zu sein, daß die nationale Sprache der Dreh- und Angelpunkt jeder nationalen kulturellen Entwicklung sein wird.

Die Erkenntnis, daß sowohl die Initiierung und Vermittlung von Lernprozessen (durch eine Lehrperson) wie auch der Vollzug des Lernprozesses durch den Lernenden, nur über eine effektive Versprachlichung erfolgen kann, hat die Erziehung zur Sprachlichkeit zum Kernstück jedes Erziehungskonzepts werden lassen. Dies gilt sowohl für die informellen traditionellen Erziehungssysteme, wie auch für das formale Bildungssystem mit einem gegliederten Schulwesen. Der deutsche Pädagoge und Philosoph Friedrich Bollnow ist dieser Hinwendung zur Sprache nachgegangen:

Die Sprache ist nicht mehr nur Gegenstand eines Unterrichtsfaches neben anderen, des Sprachunterrichts und der Sprachpflege, sondern rückt in den Mittelpunkt der gesamten Erziehung. Indem man den Menschen zur Sprache erweckt, indem man ihn reden lehrt, bildet man ihn zum Menschen. Die Pädagogik der Sprache ist also ein grundlegendes Stück einer anthropologisch orientierten Pädagogik ... Es geht nicht 
darum, den Menschen durch das Gespräch zu erziehen, sondern zugleich darum, ihn allererst zum Gespräch, zur Fähigkeit und zur echten Bereitschaft des echten Gespräches zu erziehen, weil sich darin allein das Wesen des Menschen vollenden kann. Es geht allgemein nicht darum, die Sprache als Mittel der Erziehung richtig zu begreifen und anzuwenden, sondern zugleich darum, die Sprache im Menschen als notwendiges Glied einer Selbstwerdung und seines Weltverständnisses zu entwickeln; mit einem Wort: ihn zur Sprachlichkeit zu erziehen. ${ }^{8}$

Dem kann man ein Zitat des afrikanischen Philosophen und Historikers Ki Zerbo aus seiner Geschichte Afrikas gegenüberstellen:

Der Afrikaner muß sich selbst sprechen hören können, um zu spüren, daß er wirklich lebt. Es ist Teil seiner Existenz, daß er spricht. ${ }^{9}$

Auf den ersten Blick mögen diese beiden Zitate nahezu gleich klingen, aber sie beinhalten doch zwei grundverschiedene Auffassungen von Sprache, sie beziehen sich auf zwei verschiedene Sprachen. Man kann Bollnows anthropologischen Ansatz von der Funktion der Sprache noch durch die behaviouristischen Konzeptionen der Soziolinguisten ergänzen, die nachgewiesen haben, daß der Heranwachsende, indem er die Sprache von seiner unmittelbaren Umgebung erlernt, zugleich auch die organisatorische Struktur seiner Umwelt aufnimmt, wie sie ihm in seiner Sprache vorgegeben wird, sowohl bezüglich der faktisch-materiellen Organisation wie auch bezüglich der Formen sozialer Interaktion. Sprache beinhaltet daher sowohl Offenheit wie auch Beschränkung, da sie einerseits die Mittel zur Erfassung der Umwelt bereitstellt, andererseits aber die Perspektive dieser Umwelterfassung festlegt durch die vorgegebenen Sprachstrukturen. Dadurch wird die Fähigkeit eingeschränkt, Probleme zu erfassen, die in der ursprünglich gegebenen Umwelt unbekannt sind.

In einer Gesellschaft, in der verschiedene soziale Schichten vorhanden sind und gleichzeitig damit verbundene Unterschiede hinsichtlich der Kontrolle des Zugangs zu den Statuspositionen, wobei diese Kontrolle entscheidend aufgrund von Bildung und Ausbildung ausgeübt wird, sind Kinder aus der Schicht im Vorteil, deren Angehörige über die gleichen sprachlichen Codes verfügen wie die Personen, welche die Bildungsinhalte vermitteln. Kinder aus Schichten, in denen aufgrund anderer Lebensbedingungen abweichende Sprachstrukturen entwickelt wurden, sind demgegenüber im Nachteil. ${ }^{10}$

Wenn wir Bollnows These von der zentralen Funktion der Sprache in einer anthropologisch konzipierten Pädagogik übernehmen und sie auf die Situation in Afrika übertragen, wo die Erziehung zum Teil in der einheimischen Muttersprache, zum Teil in der Fremdsprache der ehemaligen Kolonialmacht erfolgt, so wird deutlich, daß die hier geforderte Erziehung zur Sprachlichkeit gleichzeitig auch eine Erziehung zur Zwiespäl-

8 Friedrich Bollnow, Sprache und Erziehung (Stuttgart, 1964), S. 16/17.

9 Ki Zerbo, Histoire de l'Afrique noire (Paris, 1976), S. 611, deutsche Ubersetzung bei Hammer Verlag Wuppertal und als Fischer Taschenbuch.

10 Dieter Höger, Einführung in die pädagogische Psychologie (Stuttgart, 1978), S. 86. 
tigkeit sein muß, einer Zwiespältigkeit zwischen endogener und exogener Sprach- und Kulturtradition, einer Zwiespältigkeit zwischen überkommener Selbstwerdung und von außen hereingetragener Entfremdung. Wenn also Sprachlichkeit im Sinne von Einsprachlichkeit ein auch nur annähernd geschlossenes System pädagogischer Zielsetzung beinhaltet, muß Zweisprachlichkeit umgekehrt eine Rivalität, ja Unvereinbarkeit der mit der jeweiligen Sprache verbundenen pädagogischen Zielsetzung beinhalten.

Diese für ganz Afrika typische Situation, daß die allgemeine Erziehung durch das Elternhaus und die soziale Gemeinschaft in der Muttersprache, die formale Erziehung in der Schule aber in einer fremden Sprache mit fremden Werthaltungen erfolgt, wurde erstmals von der Negritude-Bewegung im "französischsprachigen" Afrika Ende der Vierziger Jahre kritisiert. Auch wenn seit der Entkolonialisierung und der Entstehung unabhängiger Staaten in Afrika gewaltige Anstrengungen unternommen wurden, die Inhalte der formalen Bildung in den Schulen zu "afrikanisieren", d. h. sie den Bedürfnissen und Gegebenheiten Afrikas anzupassen, so hat sich dennoch an der grundsätzlichen Situation bis heute nichts geändert, nämlich der Entfremdung des gebildeten Afrikaners von seiner bodenständigen kulturellen Tradition durch das Unterrichtssystem und die Unterrichtssprache in Schule und Universität. Und innerhalb dieses Systems bildete der naturwissenschaftlich-technische Unterricht sicherlich den solidesten Block der Entf remdung. Denn "Science" wird im Bewußtsein von Lehrern, Schülern und Eltern als Unterrichtsgegenstand in seinen Inhalten, Zielen, Methoden, vor allem aber in der Unterrichtssprache nirgendwo mit endogener Kulturentwicklung assoziiert, sondern ausschließlich als Kulturimport verstanden, zwar unvermeidlich, aber dennoch fremd.

In diesem Zusammenhang kommt der Entscheidung der nigerianischen Regierung von 1979 allergrößte Bedeutung für die längst überfällige Veränderung der pädagogischen Landschaft in Afrika zu, noch im Laufe dieses Jahrzehnts Englisch als Unterrichtssprache in den Schulen durch die drei Hauptsprachen Nigerias, Hausa, Yoruba und Igbo abzulösen. In Zukunft sollen also die nigerianischen Kinder nicht nur in der Grundschule, sondern während ihrer ganzen Ausbildungszeit in ihrer Muttersprache unterrichtet werden und dazu eine der beiden anderen Hauptsprachen Nigerias als Pflichtfremdsprache erlernen. Damit wird das Englische in seiner bisherigen Rolle als gesamtnigerianisches Verständigungsmittel abgelöst und auf eine Funktion als internationales Verständigungsmittel reduziert. Daß die Durchführung dieses Vorhabens mit der damit verbundenen Erstellung neuer Lehrwerke und der Ausbildung der Lehrkräfte mit einem erheblichen Kosten- und Zeitaufwand verbunden sein wird, ist klar. Außerdem wird auch mit Widerständen der zahlreichen kleineren Sprachgruppen zu rechnen sein. Aber die Signalwirkung, die von dieser Entscheidung ausgeht, ist bereits jetzt in den anderen afrikanischen Staaten zu spüren.

Nigeria hat sich durch die Aufnahme dieser Absichtserklärung zur Sprachenpolitik in seine zivile Verfassung von 1979 weiter vorgewagt als jeder andere afrikanische Staat inzwischen ist ja durch den neuerlichen Militärputsch diese Verfassung wieder außer Kraft gesetzt worden. Nigeria hatte aber auch den größten Handlungsbedarf - bei insgesamt et wa 400 verschiedenen Sprachen in seinem Staatsgebiet - aber auch den größten 
finanziellen Handlungsspielraum in der Zeit, als die Petrodollars noch reichlich flossen. Das National Language Centre sollte die Voraussetzungen der Orthographie und Bestandsaufnahme einheimischer Sprachen schaffen. ${ }^{11}$ Paradoxerweise wird aber auch im Rahmen der offiziellen Sprachenpolitik die Verbesserung des Englisch-Unterrichts gefordert und die Einrichtung eines Englisch Language Centre vorgeschlagen. Genauso, wie für die Einführung der afrikanischen Sprachen als Unterrichtssprachen argumentiert wird, so wird auch für die Verbesserung des Englisch-Unterrichts die Bedeutung dieser Sprache für die wirtschaftliche und soziale Entwicklung angeführt. ${ }^{12}$ Auch das ist nur scheinbar ein Widerspruch, denn in der nigerianischen Sprachenpolitik werden den nationalen, den regionalen, den lokalen Sprachen und der internationalen Sprache Englisch unterschiedliche Funktionsbereiche zugewiesen. Das Englische, das zumindest für den Süden Nigerias eine überregionale Lingua Franca-Funktion hat - so wie im Norden das Hausa -, soll demnach weitgehend auf eine Funktion als internationale Verkehrssprache, allenfalls auch als internationale Wissenschaftssprache beschränkt werden.

Noch vor der Verankerung dieser neuen Sprachen- und Bildungspolitik in der Verfassung fand eine erste Erprobungsphase mit dem sogenannten Ife-Projekt oder SYPPProjekt statt. In und um die Yoruba-Stadt Ife wurde dieses Six Years Primary Project folgendermaßen angelegt: Während der gesamten sechsjährigen Dauer der Grundschulausbildung wird Yoruba als Unterrichtssprache auch in den naturwissenschaftlichtechnischen Fächern eingesetzt. Englisch wird ausschließlich als Fremdsprache unterrichtet. Demgegenüber stehen die Kontrollgruppen des konventionellen Grundschulplanes, wo die ersten drei Jahre in der afrikanischen Sprache, ab dem vierten Schuljahr jedoch in Englisch unterrichtet wird. Man hatte wohl erwartet, daß die Leistungen der in Yoruba unterrichteten Schüler in den Sachkundefächern erheblich ansteigen, in Englisch dagegen abfallen würden. Dies hat sich nicht in dem erwarteten Umfang bestätigt. Die Leistungen in den Sachfächern waren zwar besser als die der in Englisch unterrichteten Kontrollgruppen, aber nicht so markant besser wie erhofft. (Mein Kenntnisstand zur Auswertung des Projekts ist inzwischen drei Jahre alt, damals war man noch auf der Suche nach plausiblen Begründungen für diesen Befund.) ${ }^{13}$ Drei Erklärungen für den hinter den Erwartungen zurückbleibenden Erfolg des Projekts bieten sich an:

1. Das Lehrpersonal war nicht ausreichend ausgebildet im Unterricht in einer afrikanischen Sprache und verfügte über keine Unterrichtserfahrung mit Yoruba.

2. Die Unterrichts- und Lehrmaterialien, die im Laufe des Projekts ständig nachgebessert wurden, waren noch nicht ausgereift, eben auch in einer Erprobungsphase.

3. Der Lehrplan war sowohl in seinen Inhalten wie auch in den Methoden und Zielsetzungen beibehalten und lediglich in eine andere Unterrichtsphase umgesetzt worden.

11 Adaralegbe (Hg) A Philosophy of Nigerian Education (Lagos, 1971).

12 Conrad M. B. Brann, The Implementation of Language Planning in Nigeria, 6th World Congress on Applied Linguistics Lund, August 1981).

13 Juliet I. Macauley, Teaching Foreign and Second Languages to the very Young - Paper presented to the WAMLA Conference on the Teaching of Foreign Languages in Ibadan, August 24-29, 1981. 
Hieraus können sich grundsätzliche Schwierigkeiten in der Versprachlichung und der sprachlichen Umsetzung der Lehrinhalte ergeben haben, die auf die in 2. genannten Lehrmaterialien und das in 1. erwähnte Lehrerverhalten, vor allem auf das Sprachverhalten des Lehrers durchgeschlagen haben. Offensichtlich wurden die faktischen Lehrinhalte an den Anfang der Uberlegungen gestellt und dann die sprachliche Umsetzung und didaktische Vermittlung rein als Instrumentalisierung angehängt, anstatt die Möglichkeiten der Versprachlichung vorrangig oder gleichrangig mit den zu vermittelnden Inhalten anzusetzen.

Eine Uberraschung erbrachte das Ife-SYPP-Projekt im sprachlichen Bereich. Die Schüler, die in Englisch nur als Fremdsprache unterrichtet wurden - also weit weniger Sprachkontakt in Englisch hatten als die Kontrollgruppen des herkömmlichen Systems -, waren in ihren Sprachleistungen nicht schlechter, in englischer Rechtschreibung sogar besser als die Kontrollgruppen.

Eine Folgerung, die man aus dem Ife-Projekt ziehen kann, ist, daß die Einführung einer af rikanischen Sprache als Unterrichtssprache allein noch keine Verbesserung der Qualität und der Effizienz des naturwissenschaftlichen Unterrichts erbringt. Die Sprache ist zwar eine wichtige Voraussetzung für die psychologische und mentale Besitzergreifung, die aber ohne inhaltliche und methodische Begleitmaßnahmen nicht wirklich vollzogen werden kann. Souleymane Diagne spricht in seinem Vortrag vor der UNESCOKonferenz in Kingston von der Psychologie der unwissenschaftlichen Gesellschaften und diagnostiziert ein Parallelphänomen zu dem Fanon'schen Kolonialismus-Syndrom: In diesen Gesellschaften herrsche - so Diagne - ein Gefühl der Unterlegenheit gegenüber der wissenschaftlich-technischen Welt, das zu Unsicherheit im Umgang mit Wissenschaft und Technik führe und Zweifel, ja sogar Angst erwecke, ob Wissenschaft und Technik überhaupt in die eigene Kultur integriert werden könne. Daraus resultiere ein Hang zur Mystifizierung der Technik, zur stummen Bewunderung aber auch zum Neid. Mystifizierung jedoch heißt Ausgrenzung des Nicht-zu-Bewältigenden aus der Alltäglichkeit.

Zwei Wege werden vorgeschlagen, Wissenschaft und Technik aus dieser Position der Unnahbarkeit zu lösen. Einmal könne eine Betrachtung der Wissenschaftsgeschichte aufzeigen, wie wissenschaftliche Konzepte und Theorien entstehen, sich durchsetzen und wieder abgelöst werden. D. h. die Relativität von Wissenschaft müsse verständlich werden und der Schleier der angeblichen Unfehlbarkeit fallen. ${ }^{14}$ Als zweiter Weg wird vorgeschlagen, an die endogenen Technologien in Afrika anzuknüpfen und von dem bereits vorhandenen technischen Know-How die Brücke zu den westlich-industriellen Technologien zu schlagen.

Die Entzauberung der Wissenschaft durch die Wissenschaftsgeschichte wird allenfalls in der Lehrerausbildung zum Tragen kommen können. Dagegen sind endogene Technolo-

14 Vgl. dazu Diagne, a.a.O., Pai Obanya, Sience and Technology in African Education, EDUAFRICA (1983), S. 74; O. J. Jegede, D. P. Brown, Primary Science Teaching (Ibadan, Lagos, 1980), S. 5/6; Israel Katoke, Culture and Development, EDUCAFRICA, 7 (1981), $125 \mathrm{f}$. 
gien vor allem in ländlichen Bereichen noch häufig zu beobachten. Ich möchte zwei Beispiele anführen:

In ganz Afrika gibt es eine lange Tradition der Eisenverhüttung. Einige dieser Hochöfen waren bis vor kurzem oder sind noch bis heute in Betrieb. Ich selbst hatte Gelegenheit, einen solchen Hochofen zu besichtigen und einen Eindruck von der Gesamtanlage zu erhalten, d. h. den Erzabbaustellen (im Tagebau), den Kohlenmeilern und dem Köhlerreiprozeß, der Auswahl der Erze, dem Schmelzvorgang mit seinen Vorkehrungen zur Kontrolle der Schmelztemperatur durch ein fein dosierbares System der Sauerstoffzufuhr in den Verbrennungsraum. Der Hochofenmeister, inzwischen ein älterer Herr um die 70, sprach kein Wort der Wissenschaftssprache Englisch, sondern nur Yoruba und vermochte dennoch den gesamten Schmelzvorgang zu erklären. Der gesamte Herstellungsprozeß ist in Yoruba verbalisierbar und damit auch vermittelbar. Allerdings beruhen die Beurteilungen und Entscheidungen z. B. über die Qualität des Erzes, der Kohle, der Temperaturregulierungen auf langjähriger Erfahrung, nicht auf Meßdaten, Analysen.

Zweites Beispiel: In der nordnigerianischen Stadt Jos wurde ein Freilichtmuseum gebaut, das u. a. Nachbauten der afrikanischen Lehmarchitektur aufweist. Die Bauten wurden alle mit den traditionellen Bautechniken erstellt; hier also mit luftgetrockneten Ziegeln, verbunden mit einem Lehmmörtel und mit einem Lehmglattstrich verputzt. Zuletzt wird die Außenhaut der Gebäude mit einem Imprägnieranstrich versehen, der durch Ansetzen der Rinde eines bestimmten Baumes in Wasser gewonnen wird. Dieser Imprägnieranstrich erhöht die Lebensdauer des Außenputzes um 5 bis 6 Jahre. Dabei muß berücksichtigt werden, daß während der Regenzeit, aber auch durch extreme Hitze und Trockenheit, der Außenputz von Gebäuden extremen Belastungen ausgesetzt ist. Wie mir mein Informant berichtete, wurde im wissenschaftlichen Begleitprogramm beim Aufbau des Museums eine chemische Analyse des Imprägnieranstrichs durchgeführt, die jedoch keine restlose Aufklärung über die Wirksubstanzen erbracht habe. Auch hier haben wir es wiederum mit vorwissenschaftlicher Technologie zu tun, mit reiner Erfahrungstechnologie, deren Verfahrensweisen ausschließlich durch Erprobung über lange Zeiträume hinweg gewonnen wurden, nicht jedoch durch systematisches analytisches Deduzieren aus Kausalzusammenhängen und darauf begründetes Projezieren und Prognostizieren von Wirkungen und Ergebnissen. Die Erfahrungstechnologie ist überwiegend reproduzierend, undynamisch und allenfalls durch Zufallsentdeckungen kann sie innovativ werden, im Gegensatz zur zielorientierten wissenschaftlichen Vorgehensweise.

Für den naturwissenschaftlich-technischen Unterricht hat das Anknüpfen an endogenen Technologien den Vorteil, daß die Versprachlichung in der afrikanischen Sprache unbestritten ist. Die ganze Diskussion, ob die afrikanische Sprache die erforderliche Lexik und Begrifflichkeit verfügbar hat, stellt sich nicht. Denn diese Technologien sind Teil der Kultur, sind Ausdruck dieser Kultur, genauso wie die Sprache, und werden in genau dieser Sprache auch ausgedrückt.

Das Anknüpfen an endogene Technologien empfiehlt sich insbesondere für den Anfangs- 
unterricht, wo das Beobachten und Beschreiben von Vorgängen oder Phänomen im Mittelpunkt steht. Es ist of fenkundig, daß die Beschreibung eines Vorgangs, der Teil der sozialen und kulturellen Umwelt ist, von einem Schüler leichter zu bewältigen ist, als die Beschreibung einer Versuchsanordnung mit Laborcharakter, dessen Bezug zur eigenen Wirklichkeit und Umwelt durch seine Abstraktion und Künstlichkeit überhaupt nicht erkannt werden kann. Bisweilen mag es sogar gelingen, eine Brücke von traditioneller Technologie zu industrieller Technologie zu schlagen: z. B. die Verwendung von Stroh in den einzelnen Lehmziegeln; das Einarbeiten von Zweigen und Balken in tragende Teile der Lehmarchitektur erfüllen ähnliche Funktionen wie die Armierung bei der Betonbauweise.

Der Schritt von endogener zu moderner Technologie wird sicherlich der schwierigste Teil der Vermittlung im Unterricht sein. Andererseits kann die industrielle Technologie im Sinne der Groß-Technologie ja gar nicht Gegenstand eines Grundunterrichts sein. Vielmehr geht es ja hier "nur" um das Beobachten, Erkennen und Beschreiben elementarer Vorgänge.

Die Hinwendung zu traditionellen Technologien als Gegenstand und Vorbild für den wissenschaftlich-technischen Unterricht im Grundschul- und unteren Sekundarschulbereich ist signifikant für die pädagogische und bildungspolitische Diskussion. Daß man den Weg zur industriellen Technologie nun eher über den historischen Umweg der traditionellen Technologie sucht, zeigt einen Aspekt der Entzauberung von GroßTechnologie, wie sie Diagne fordert, sie zeigt aber auch eine Desillusionierung der Modernisierungsträume und eine realistischere, pragmatische Haltung sowohl zu einer integrierten, als auch der Kultur und Gesellschaft angepaßten Technologie und zu einer effektiveren, weil angepaßten Form der unterrichtlichen Vermittlung.

Die zweite wesentliche Änderung in der neueren pädagogischen Diskussion über den naturwissenschaftlich-technischen Unterricht betrifft eine grundsätzliche Umorientierung der Lehrmethoden und der Unterrichtspraxis. Bisher bestand Unterricht meist im schlichten Darlegen von Fakten und Sachverhalten und dem Memorieren; ohne Schülerbeteiligung und weitgehend ohne experimentelle Arbeit. Die Gründe dazu liegen in der mangelnden Ausbildung der Fachlehrer und dem Fehlen von Laborausstattungen und Laborpersonal. Gerade dieses Darlegen und Memorieren hat die Verwendung von englischen Fachausdrücken begünstigt, d. h. die Verwendung eines nicht verstandenen und nicht-integrierten Sprachmaterials, das einen wirklichen Lernerfolg beinahe unmöglich macht.

Die Arbeiten aus jüngerer Zeit fordern einhellig einen schülerorientierten experimentellen Unterricht, der seine Problemstellung und Materialien aus der unmittelbaren Umgebung der Schüler bezieht, insbesondere auch land- und hauswirtschaftliche Probleme einbezieht. ${ }^{15}$ Die Abwendung von kognitivem Drill und die Hinwendung zu entdecken-

15 Pai Obanya, Science and Technology in African Education, EDUCAFRICA (1983), 68/69; D. Fall, Current Trends in Physical Science Teaching in Senegal EDUCAFRICA 7 (1981), 199 f.; Udo Bude, Primarschulen und ländliche Entwicklung DSE (Bonn, Sept. 1983); Vorträge von Dr. Ralph N. Oranu, R.C. Ebene u. a. m. 
dem Lernen setzt eher weniger als mehr apparative Ausstattung in den Schulen voraus, aber sie erfordert eine souveränere, einfallsreiche und improvisierfreudige Lehrerpersönlichkeit, was die gegenwärtige Lehrerausbildung (auch wegen der Unterrepräsentanz der naturwissenschaftlichen Fachlehrer) nicht in ausreichendem Maße leistet. Der integrierte Naturkundeunterricht soll vor allem die Beobachtungsgabe der Schüler schulen. Dem soll ab dem 3. Schuljahr eine Phase der Formulierung von ersten Verallgemeinerungen über die gemachten Beobachtungen folgen. Das Deduzieren elementarer Lehrsätze aus Beobachtungen oder Experimenten soll Teil des Unterrichts in der Sekundarstufe sein.

Auch hier wird wiederum deutlich, wie wichtig eine gründliche Sprachausbildung für einen erfolgreichen Sachkundeunterricht ist: zum einen können natürlich die gemachten Beobachtungen, da sie ja alle aus dem unmittelbaren Umgebungsbereich stammen, sehr wohl in der afrikanischen Muttersprache verbalisiert werden. Zugleich stellt aber die (zum Teil freie) Verbalisierung an den Schüler höhere sprachliche Anforderungen als das Repetieren von englischen Fachausdrücken. Die sprachliche Begriffsbildung und die Erprobung der eigenen Fähigkeit zur Anwendung dieser Begrifflichkeit anhand der naturkundlichen Beobachtungen geht Hand in Hand. Sprachunterricht und Naturkunde sind ebenfalls integriert. Die entscheidende Neuerung dieser Integration von Naturkunde und Sprachbildung ist jedoch, daß dabei die operativen Fähigkeiten der Schüler trainiert werden: Probleme und Fragen aus der unmittelbaren Schülerumwelt werden aufgegriffen, Lösungswege erprobt, Strategien zur Problemlösung entwickelt und sprachlich umgesetzt, also alles Fähigkeiten, die beim kognitiven Drill nicht entwickelt wurden, die aber für die Persönlichkeitsentwicklung unabdingbar sind. So werden dem naturwissenschaftlich-technischen Unterricht über die fachspezifischen Kenntnisse und über die Hoffnungen, Modernisierung und Entwicklung voranzutreiben, noch sehr weitgefaßte allgemeine Lehrziele auferlegt, eben die Fähigkeit zur Problemlösung.

O. J. Jegede und P. D. Brown umreißen die Ziele des naturwissenschaftlichen Unterrichts in der Grundschule wie folgt:

By teaching children science and about the changes that have taken place as a result of science and technology, the idea of change is more understandable and hence more acceptable. In this way future change which will help to improve the standard of living for the average Nigerian will be seen for what they are and not as forces causing the erosion of national culture. ${ }^{16}$

Das gewichtigste Argument der früheren pädagogischen Diskussion in Afrika, daß der naturwissenschaftlich-technische Unterricht die Grundvoraussetzung für wirtschaftlichen und sozialen Fortschritt sei, steht für die beiden Autoren überhaupt nicht mehr zur Debatte. Die Auswirkungen von Wissenschaft und Technik sind nicht aufzuhalten, genauso wenig wie deren Rückwirkungen auf Kultur und Gesellschaft. Aber - und hier

auf 2nd International Conference on Teaching Practice and Teacher Effectiveness, University of Nigeria Nsukka, March 1983; Nalletamby, a.a.O.

16 O. J. Jegede, D. P. Brown, Primary Science Teaching, S. 7 f. 
kommt eine ganz neue Dimension in die pädagogische Diskussion - das Hauptziel des naturwissenschaftlich-technischen Unterrichts wird hier nicht mehr nur in der Beherrschung des Stoffes gesehen, sondern zugleich in der Beherrschung und Bewältigung der sozialen und kulturellen Folgeerscheinungen des wissenschaftlich-technischen Wandels. Der Begriff der "integrated science" wird also durchaus ernst genommen in allen seinen Bedeutungen: Nicht nur die einzelnen naturwissenschaftlichen Fächer sollen untereinander integriert sein, auch das Konzept der Wissenschaft per se soll in die Kultur und Gesellschaft integriert werden. 


\title{
Presumption of Marriage in Tanzania
}

\author{
By B. A. Rwezaura
}

Section 160 of the Tanzania Law of Marriage Act provides that where it is proved that a couple has cohabited as husband and wife for at least two years, there shall be a rebuttable presumption that the two are married. Where such presumption is rebutted, the woman shall be entitled to apply to the court for an order of maintenance (and other reliefs) for herself and any children of the runion'. The interpretation which courts have given to the above section is not clear and could lead to confusion. This is so mainly because courts have not drawn distinction between two different types of presumption, i. e. the first one dealing with long cohabitation where parties have not gone through any ceremony of marriage (section 160) and the second where parties have gone through a ceremony of marriage and the presumption relates only to the validity of that ceremony. This second type of presumption is covered under section 41 of the Act. Also judicial interpretation of section 160 has given the impression that this section can be applied to cases where parties had no initial capacity to marry. This interpretation is not legally tenable either because the law cannot presume against ifself. Unfortunately this opinion has the effect of denying financial benefits to many women cohabitees and their children but the wording of the section does not permit any other interpretation. Finally, given the fact that today a number of people are cohabiting without ceremony, the Act ought to provide for registration of such unions after the requirements of section 160 have been satisfied.

\section{Natural Sciences, Technology and Language Instruction in Africa}

\section{By Eckard Breitinger}

In a series of conferences the newly independent African nations have defined their educational policies, hoping to enhance economic growth by improving educational facilities. It was generally agreed that the inherited educational systems were not adequate to meet the needs of the new nations. The humanities and social science programmes were the first to be Africanized. 'By contrast, science and technology were for a long time considered culturally indifferent. However, the combination of science as a foreign concept of thought on the one hand and of foreign languages of instruction' on the other hand have obviously been at the root of the ineffectiveness of science education. Although a new emphasis was placed, in educational policies, on the teaching of and in national African languages and on African natural cultures, this still left science education in a glaringly isolated position within school syllabi, as the one and major domain of alienation of African students, due to the alienness of the subject matter, and of the method 
and linguistic medium of instruction. It is suggested that mastery of science and technology can only be achieved if scientific and technological concepts can be mastered linguistically and thereby, conceptually. Appropriate technology must mean that the recipients are able to grasp it culturally, socially and linguistically. This could be achieved through instruction of basic science based on traditional African technology. Indigenous technological traditions are part of the national culture and, as such, can be verbalized and taught in national languages, i. e., in the linguistic media that determine intellectual conceptualization on the part of the students Integrated science thus comes to mean that scientific concepts are integrated into the entire school syllabus, including language teaching as the means to comprehend scientific concepts linguistically, and also that science, through the aid of the national language, becomes integrated into the cultural and social environment.

\section{Adjustment of Structural Change in Crisis Management Policies of Tanzania}

\section{By Thomas J. Heinrich}

Recent development in Tanzania is but one example of a recurring feature: a developing country in a structural economic crisis exposed to coercion and pressures by aid donor countries and the World Bank group. As a first step the article briefly examines two controversial viewpoints on the causes of the current crisis in Tanzania. Comments presenting the crisis as mainly due to inadequate internal policies in Tanzania are rejected and interpreted as part of the initially mentioned pressures.

The example of Tanzania is most relevant since an autochthonous type of socialist development quite different from the concepts of most developed countries seems to be at stake. However, does the "Tanzanian Model" exist any longer? Some elements of Ujamaa socialism still persist but, under prevailing external constraints, is Tanzania free to defend the basis of "Ujamaa"? The 'Structural Adjustment Programmer of Tanzania (SAP), adopted in 1982 as an intermediate 'for crisis management ' development plan, is taken as an example to approach this issue. For this purpose the remedies' prescribed to the Tanzanian patient by the IMF and the IBRD are summarized and their underlying intentions exposed. Structural adjustment as spelt out by the World Bank group rather means a structural transformation of Tanzanian politics: integration into the world market, less state intervention in the economy. Three theses are put forward to discuss the SAP and the range of options open to Tanzania to maintain a self reliant strategy: the freedom to choose has become very narrow. However, there remains a fairly significant margin for action: exercising influence on the selection and design of development projects: This option is not taken seriously by the Tanzanian government. In her short history, Tanzania was never in an economic position to develop an adequate national infrastructure on which to build the 'Tanzanian Model. Thus, lacking the material foun- 